\title{
MR-MPS-SW e CERTICS: Mapeamento e Diretrizes para a Implantação Conjunta dos Modelos
}

\author{
Larissa Lopes de Araujo ${ }^{1,2}$, Ana Regina Rocha ${ }^{1}$, Gleison Santos ${ }^{3}$ \\ ${ }^{1}$ COPPE/UFRJ - Universidade Federal do Rio de Janeiro - Caixa Postal 68511 - \\ CEP 21.941-972 - Rio de Janeiro, RJ \\ ${ }^{2}$ ECO Sistemas - Rua Dom Gerardo, $\mathrm{n}^{\circ} 35-9^{\circ}$ e $10^{\circ}$ andar - Centro \\ CEP 20.090-030 - Rio de Janeiro - RJ \\ ${ }^{3}$ Programa de Pós-Graduação em Informática - Universidade Federal do Estado do Rio \\ de Janeiro (UNIRIO) - Av. Pasteur 458, Urca, CEP 22.290-240 - Rio de Janeiro, RJ \\ darocha@cos.ufrj.br, larissa.araujo@ecosistemas.com.br, \\ gleison. santosduniriotec.br
}

\begin{abstract}
The Information Technology industry growth in Brazil has stimulated organizations to invest in software process improvement to achieve higher quality in their products and also to obtain certifications required in public and international competitions. Multimodels implementations require great knowledge on the involved models and their intersections to reduce effort, costs and also processes redundancy. This paper presents the results of a systematic mapping study on harmonizing multimodels. We also present the mapping of MR-MPS-SW models and CERTICS software certification, including adherence guidance, which allows supporting organizations that want to implement them together.
\end{abstract}

Resumo. O crescimento da indústria de Tecnologia da Informação no Brasil vem impulsionando o investimento das organizações em melhoria de processos de software, com o propósito de alcançar maior qualidade em seus produtos, além de obter as qualificações requeridas para concorrências públicas e internacionais. A utilização de multimodelos exige grande conhecimento destes modelos e suas interseções para que a implementação conjunta não gere processos redundantes, bem como esforços e custos desnecessários. Este artigo apresenta o resultado de um mapeamento sistemático sobre harmonização de multimodelos e também apresenta o mapeamento realizado entre o modelos MR-MPS-SW e a certificação de software CERTICS, com a orientação da aderência, visando apoiar as organizações que queiram implementá-los em conjunto.

\section{Introdução}

O crescimento da globalização seguido pela competição do mercado internacional tem impulsionado as organizações por contínuas inovações e melhorias de processos e produtos (BALDASSARRE et al., 2010). As organizações realizam investimentos em melhoria de processos, com o propósito de alcançar maior qualidade em seus produtos, além de obter as qualificações requeridas em concorrências públicas e internacionais.

Modelos como CMMI (SEI, 2010), ITIL (itSMF UK, 2012), ISO/IEC 15504-2 (ISO/IEC, 2004) e Six-Sigma (TENNANT, 2001) estão entre as técnicas ou modelos de processos mais utilizados (PARDO et al., 2011a). Pardo et al. (2011a) apresentam como os modelos específicos apoiam as múltiplas necessidades de Tecnologia da Informação 
(TI), como o desenvolvimento de software, a manutenção, a operação, a segurança de TI e a governança de TI. Embora haja diversos modelos, seria imprudente acreditar que qualquer modelo definido até o momento possui uma solução completa para o gerenciamento dos processos no contexto da TI.

Com mais de 10 anos de existência e com 649 avaliações realizadas até Junho de 2015 (SOFTEX, 2015), o Modelo de Referência MR-MPS-SW (SOFTEX, 2012a) é hoje um modelo consolidado no Brasil e que está iniciando sua difusão na América Latina, especialmente na Colômbia, Argentina, Uruguai, México e Peru, com 6 avaliações internacionais realizadas. Seu objetivo é apoiar a melhoria de processos de software, principalmente, em pequenas e médias organizações de software.

O CERTICS (CTI Renato Archer, 2013), modelo de certificação de software publicado em 2013, tem por objetivo comprovar se um software é resultado de desenvolvimento e inovação tecnológica no Brasil. A sua relevância no cenário das empresas fornecedoras de Tecnologia da Informação é demonstrar que o seu software é resultante de desenvolvimento e inovação tecnológica no País, construído com competência tecnológica e melhoria contínua, à partir de ações de monitoramento do mercado e das necessidades dos clientes. Este modelo vem despertando interesse nas organizações, muitas delas já avaliadas no MR-MPS-SW. Segundo o site da CTI (CTI Renato Archer, 2015), até Setembro de 2015, 27 softwares haviam recebido a certificação CERTICS.

A utilização de ambientes multimodelos exige grande conhecimento destes modelos e de suas interseções para que a implementação conjunta não gere processos redundantes, bem como esforços e custos desnecessários. Considerando a dificuldade da implantação de múltiplos modelos e o interesse pelos modelos MR-MPS-SW e CERTICS, este artigo apresenta o mapeamento destes modelos, buscando identificar o grau de cobertura do MR-MPS-SW, considerando seus diversos níveis, com relação aos resultados esperados pelas áreas de competência do CERTICS. Uma versão resumida deste mapeamento foi apresentado no WAMPS 2014 (ARAUJO et al., 2014). No presente trabalho foram estendidas as discussões relacionadas ao detalhamento dos modelos de referência, da metodologia de pesquisa, das regras de cobertura, avaliação realizada para garantir a adequação do mapeamento entre os modelos e orientações para implementação conjunta dos modelos. Além disso, é apresentado o resultado de mapeamento sistemático da literatura sobre harmonização de multimodelos.

Além desta seção, este artigo está organizado em outras 7 seções. Na seção 2, são apresentadas as principais questões relacionadas à melhoria de processos multimodelos. A seção 3 apresenta o mapeamento sistemático da literatura. A seção 4 descreve os modelos MR-MPS-SW e CERTICS. A seção 5 apresenta a metodologia de pesquisa utilizada para a elaboração do trabalho, incluindo como o mapeamento foi realizado e o resultado da revisão por pares conduzida para garantir a adequação dos resultados obtidos. A cobertura do MPS-SW com relação ao CERTICS, juntamente com as orientações da implantação em conjunto está na seção 6. Por fim, na seção 7 são apresentadas as considerações finais.

\section{Melhoria de Processos Multimodelos}

Segundo Pardo (2012), alguns fatores podem influenciar a utilização de múltiplos modelos, como nichos de mercado de modelos específicos, melhoria de práticas de 
processos legados, posicionamento de negócio, alavancadas ou incorporação societária, melhoria da capacidade dos processos e crescimento do negócio. Entretanto, o uso simultâneo de múltiplas abordagens pode trazer problemas, entre os quais a identificação e manipulação de diferentes abordagens com diferentes estruturas, granularidade, terminologia, conteúdo, tamanho e complexidade (KELEMEN, 2013). Estes problemas podem gerar esforço e custo extras, além de aumentar o risco de ineficiências e redundâncias (BALDASSARRE et al., 2010). Como possível solução para estes problemas, devem-se realizar esforços de harmonização, para identificar interseções e partes comuns, criando uma solução de melhoria multimodelos.

O SEI (Software Engineering Institute) descreve através de seu ambiente de melhoria de processos multimodelos (PriMe) a construção de uma estrutura de melhoria de Processo na Organização (SIVIY et al., 2008). Esta orientação sugere algumas etapas para a implantação de multimodelos, como: (i) alinhamento dos objetivos organizacionais e de melhoria dos modelos pretendidos, incluindo a identificação de cada melhoria candidata; (ii) definição estratégica das melhorias de tecnologia a serem implantadas, entendendo as sobreposições dos modelos; (iii) detalhamento da tecnologia e arquitetura do processo a ser implantados; (iv) implementação de sua solução de melhoria de processos multimodelos; e (v) medição dos resultados. Esta é uma proposta de harmonização de múltiplos modelos.

Em relação à integração de modelos, FERREIRA et al. (2011) apresentam um modelo conceitual para o gerenciamento das informações para apoiar nas auditorias e avaliações multimodelos. Segundo estes autores um primeiro modelo deve ser implementado. O segundo deve observar as questões para completar a implantação com o primeiro, pois harmonizar significa encontrar similaridades e sinergias que facilitem a implantação conjunta.

No que se refere ao mapeamento de dois ou mais modelos, vários trabalhos vêm sendo realizados nesta direção. CATER-STEEL (2006) harmoniza CobiT, ITIL, ISO 9000 e CMMI. BALDASSARRE et al. $(2010 ;$ 2011) harmoniza CMMI com ISO 9001. O CMMI v1.2 é harmonizado com ISO/IEC 15504-2 por diversos autores como PINO (2009) e PELDZIUS e RAGAISIS (2011). MELLO (2011) harmoniza os modelos MRMPS-SW e CMMI-DEV. A SOFTEX publicou, ainda, outros dois mapeamentos, do MR-MPS-SW em relação à NBR ISO/IEC 29110-4-1:2012 (SOFTEX, 2012b) e do MR-MPS-SW em relação ao modelo MoProSoft (2012c).

\section{Mapeamento Sistemático da Literatura}

Um mapeamento sistemático da literatura foi realizado (ARAUJO, 2014) com o objetivo de identificar o estado da arte da utilização de multimodelos. A questão principal de pesquisa utilizada foi "Que abordagens, técnicas e processos têm sido propostos e/ou utilizados para mapeamento, integração e harmonização dos modelos MPS, CMMI-DEV, MPT, CERTICS e/ou ISO?'. E as questões secundárias utilizadas no estudo foram "Quais critérios têm sido propostos e/ou adotados para o mapeamento entre os modelos MPS, CMMI-DEV, MPT, CERTICS e/ou ISO" e "Quais são as características das iniciativas de melhorias de processos de software multimodelos em organizações?". Este mapeamento foi executado no contexto da dissertação de Mestrado "Mapeamento do MPS.SW com os Modelos MPT.BR e Certics". O MPT.BR, que não faz parte do escopo deste artigo, é um modelo de Melhoria do Processo de Teste 
Brasileiro (SOFTEX RECIFE, 2011).

Este mapeamento sistemático foi complementar ao realizado por Mello (2011). Foi utilizada uma adaptação à sua expressão de busca, com a complementação das palavras "MPT.Br" e "CERTICS" e restrição de datas (este estudo foca publicações de 2011 até 2014). Desta forma, a realização do mapeamento sistemático contribuiu para o entendimento do cenário atual das empresas, na perspectiva de implantação multimodelos. A estratégia de busca nas bibliotecas digitais e anais seguiram a estratégia do Mello (2011), que excluiu algumas bases em rodadas iniciais, por fim, identificou apenas a Scopus como base relevante.

A expressão de busca utilizada foi: ("software process" OR "software processes" OR "process evolution" OR "process improvement" OR "melhoria de processo" OR "evolução de processo") AND (("ISO" AND "CMMI") OR ("ISO" AND "MPS") OR ("MPS" AND "CMMI") OR ("MPT") OR ("CERTICS")) AND (("multimodels" OR "multi-models" OR "multimodel" OR "multi-model" OR "multiple tecnologies") OR ("HARMONIZING" OR "INTEGRATED" OR "COMPARING" OR "MAPPING" OR "APPLYING")).

Na primeira rodada, em fevereiro de 2014, foram encontrados 334 artigos. Estes artigos incluíam o mapeamento sistemático realizado por Mello (2011) e novos artigos publicados após 2011. Na segunda rodada, em abril de 2014, foram incluídas as palavras "CERTICS" e "MPT" na expressão de busca e a restrição de datas (entre janeiro de 2011 a março de 2014). Dos 177 artigos encontrados pela segunda rodada, foram selecionados 14 artigos que permitiram responder às questões principal e secundárias. A Tabela 1 apresenta os artigos selecionados.

Tabela 1: Artigos do Escopo do Mapeamento Sistemática (ARAUJO, 2014)

\begin{tabular}{|c|l|c|l|}
\hline ID & \multicolumn{1}{|c|}{ Autor(es) } & Ano & \multicolumn{1}{|c|}{ Título } \\
\hline 1 & $\begin{array}{l}\text { Pardo, C.; Pino, F.; Garcia, F.; } \\
\text { Velthius, M. \& Baldassarre, } \\
\text { M. }\end{array}$ & 2011 & $\begin{array}{l}\text { Trends in Harmonization of Multiple } \\
\text { Reference Models }\end{array}$ \\
\hline 2 & $\begin{array}{l}\text { Ferreira, A.; Machado, R. \& } \\
\text { Paulk, M. }\end{array}$ & 2011 & $\begin{array}{l}\text { Supporting audits and assessments in multi- } \\
\text { model environments }\end{array}$ \\
\hline 3 & $\begin{array}{l}\text { Hauck, J.; Von Wangenheim, } \\
\text { C.; Mc Caffery, F. \& } \\
\text { Buglione, L. }\end{array}$ & 2011 & $\begin{array}{l}\text { Proposing an ISO/IEC 15504-2 compliant } \\
\text { method for process capability/maturity } \\
\text { models customization }\end{array}$ \\
\hline 4 & $\begin{array}{l}\text { Pardo, C.; Pino, F.; García, F.; } \\
\text { Piattini, M. \& Baldasarre, M. }\end{array}$ & 2011 & $\begin{array}{l}\text { Supporting the combination and integration } \\
\text { of multiple standards and models }\end{array}$ \\
\hline 5 & $\begin{array}{l}\text { Peldzius, S. \& Ragaisis, S. } \\
6\end{array}$ & $\begin{array}{l}\text { Ruiz, J.; Osorio, Z.; Mejia, J.; } \\
\text { Munoz, M.; Chavez, A. \& } \\
\text { Olivares, B. }\end{array}$ & $\begin{array}{l}\text { Comparison of maturity levels in CMMI- } \\
\text { DEV and ISO/IEC 15504 }\end{array}$ \\
\hline 7 & $\begin{array}{l}\text { Baldassarre, M.; Caivano, D.; } \\
\text { Pino, F.; Piattini, M. \& } \\
\text { Visaggio, G. }\end{array}$ & $\begin{array}{l}\text { Definition of a hybrid measurement process } \\
\text { for the models ISO/IEC 15504- ISO/IEC } \\
\text { 12207:2008 and CMMI Dev 1.3 in SMEs }\end{array}$ \\
\hline 8 & $\begin{array}{l}\text { Banhesse, E.; Salviano, C. \& } \\
\text { Jino, M. }\end{array}$ & $\begin{array}{l}\text { Harmonization of ISO/IEC 9001: 2000 and } \\
\text { CMMI-DEV: From a theoretical comparison } \\
\text { to a real case application }\end{array}$ \\
\hline 9 & $\begin{array}{l}\text { Buglione, L.; Hauck, J.; Von } \\
\text { Wangenheim, C. }\end{array}$ & $\begin{array}{l}\text { Towards a metamodel for integrating } \\
\text { multiple models for process improvement }\end{array}$ \\
\hline
\end{tabular}




\begin{tabular}{|c|c|c|c|}
\hline ID & Autor(es) & Ano & Título \\
\hline & McCaffery, F. & & $\begin{array}{l}\text { Applying the LEGO approach for improving } \\
\text { estimates }\end{array}$ \\
\hline 10 & $\begin{array}{l}\text { Costa Furtado, J. \& Bezerra } \\
\text { Oliveira, S. }\end{array}$ & 2012 & $\begin{array}{l}\text { A process framework for the software and } \\
\text { related services acquisition based on the } \\
\text { CMMI-ACQ and the MPS.BR acquisition } \\
\text { guide }\end{array}$ \\
\hline 11 & $\begin{array}{l}\text { García-Mireles, G.; Moraga, } \\
\text { M.; García, F. \& Piattini, M. }\end{array}$ & 2012 & $\begin{array}{l}\text { Towards the harmonization of process and } \\
\text { product oriented software quality Sim } \\
\text { approaches }\end{array}$ \\
\hline 12 & $\begin{array}{l}\text { Pardo, C.; Pino, F.; García, F.; } \\
\text { Piattini, M. \& Baldassarre, M. }\end{array}$ & 2012 & $\begin{array}{l}\text { An ontology for the harmonization of } \\
\text { multiple standards and models }\end{array}$ \\
\hline 13 & Peldzius, S. \& Ragaisis, S. & 2012 & $\begin{array}{l}\text { Framework for usage of multiple software } \\
\text { process models }\end{array}$ \\
\hline 14 & $\begin{array}{l}\text { Garzás, J.; Pino, F.; Piattini, } \\
\text { M. \& Fernández, C. }\end{array}$ & 2013 & $\begin{array}{l}\text { A maturity model for the Spanish software } \\
\text { industry based on ISO standards }\end{array}$ \\
\hline
\end{tabular}

Dos 177 artigos encontrados pela segunda rodada, foram selecionados 14 artigos que permitiram responder às questões primárias e secundárias propostas neste estudo. Estes artigos selecionados podem ser divididos em três grandes grupos, de acordo com o seu escopo de pesquisa:

(i) Nove artigos que definem um framework e/ou ontologia:

- FERREIRA et al. (2011) apresentam um modelo conceitual para o gerenciamento das informações das metas em Qualidade, para apoiar nas auditorias e avaliações multimodelos.

- HAUCK et al. (2011) propõem um framework para customização de modelos de maturidade/capacidade de processos de software para um setor específico, tendo como base o padrão ISO/IEEE. O modelo proposto por Hauck, SPCMMs (Software Process Capability/Maturity Models), possui duas dimensões: do processo e da capacidade/maturidade.

- PARDO et al. (2011b) citam o framework apresentado anteriormente, detalhando os elementos e ferramentas utilizados para apoiar a harmonização de multimodelos de referência, considerando o crescimento dos modelos e aumento do interesse das organizações.

- BANHESSE et al. (2012) apresentam um metamodelo de capacidade de processo para uma integração dinâmica de elementos de múltiplos modelos de qualidade, durante um ciclo de melhoria do processo, utilizando o MDE (Model Driven Engineering).

- BUGLIONE et al. (2012) definem um framework chamado LEGO (Living EnGineering prOcess) com o objetivo de harmonizar dois ou mais modelos de qualidade, com áreas comuns em Engenharia de Requisitos, para obter mais informação e valor para a organização, e assim, melhorar sua estimativa organizacional.

- FURTADO et al. (2012) definem um framework para aquisição de software e serviços relacionados, voltado aos modelos CMMI-ACQ e MPS.BR Aquisição.

- PARDO et al. (2012) afirmam que as organizações precisam de grande 
empenho diante da complexidade e dificuldade para entender e interpretar alguns modelos e desenvolve uma ontologia para definir principais conceitos relacionados à harmonização de múltiplos modelos, aplicando estes conhecimentos em uma ferramenta Web que apoia esta ontologia.

- PELDZIUS et al. (2012) relatam a construção de um framework para harmonização de modelos, chamado TSPM (Transitional Software Process Model).

- GARZÁS et al. (2013) apresentam uma proposta de adaptação de alguns modelos ISO, para melhorar processo de software e obter certificação de maturidade organizacional. Para isto, desenvolvem um framework chamado AENOR, com o objetivo de melhorar o processo de software em pequenas organizações na Espanha, com menos de 50 empregados.

(ii) Quatro artigos que comparam e/ou harmonizam dois ou mais modelos:

- PELDZIUS et al. (2011) avaliaram as relações entre os níveis de maturidade do CMMI-DEV e a ISO/IEC 15504, apresentando uma proposta de mapeamento.

- RUIZ et al. (2011) propõem um processo híbrido que atenda aos requisitos das normas ISO/IEC 15504 - ISO/IEC 12207:2008 e o modelo CMMI-DEV 1.3 para pequenas e médias organizações, onde a medição vem se tornando uma necessidade, quando a pequena organização utiliza métodos ágeis.

- BALDASSARRE et al. (2011) propõem um processo de harmonização para apoiar organizações interessadas em introduzir melhorias de qualidade e práticas de desenvolvimento de software, utilizando o mapeamento da norma ISO 9001:2008 e o modelo CMMI-DEV, com a utilização do Goal Question Metrics (GQM), para a definição das metas.

- GARCIA-MIRELES et al. (2012) descrevem uma harmonização de modelos de melhoria de processo de software com uma abordagem diferenciada, com a orientação através das metas de melhoria da qualidade do produto de software.

(iii) Um artigo que realiza uma revisão sistemática da harmonização de multimodelos:

- PARDO et al. (2011a) analisam o estado da arte de iniciativas de harmonização de múltiplos modelos e apresenta um framework que orientam as organizações na utilização de vários modelos. Após realizarem este estudo sobre as tendências na utilização de múltiplos modelos de referência, PARDO et al. (2011a; 2011b) propõem uma terminologia unificada para homogenizar as diversas estruturas dos diferentes modelos e harmonizar as técnicas que podem ser utilizadas através de um framework.

Após a realização do protocolo da pesquisa foi possível responder as questão principal: "Que abordagens, técnicas e processos têm sido propostos e/ou utilizados para mapeamento, integração e harmonização dos modelos MPS, CMMI-DEV, MPT, CERTICS e/ou ISO?". As abordagens mais utilizadas para o mapeamento, integração e harmonização de modelos têm sido: (i) a criação de frameworks e ontologias orientados a modelos de maturidade; e (ii) a comparação e harmonização dos modelos. 
As abordagens mais utilizadas para o mapeamento, integração e harmonização de modelos têm sido a criação de Frameworks e Ontologias orientados a modelos de maturidade e a comparação e harmonização de processos. Entre os modelos encontrados no mapeamento estão CMMI-DEV (SEI, 2010), ISO/IEC 15504-2 (ISO/IEC, 2004), ISO/IEC 12207 (ISO/IEC, 2008a) e ISO/IEC 9001 (ISO/IEC, 2008b).

Dos 14 artigos selecionados nesta revisão, 71,43\% utilizou a abordagem de definição de framework para a realização do mapeamento dos multimodelos.

Em relação à primeira questão secundária, "Quais critérios têm sido propostos e/ou adotados para o mapeamento entre os modelos MPS, CMMI-DEV, MPT, CERTICS e/ou ISO?", 50\% dos artigos apresentaram um critério (FERREIRA et al. (2011), PARDO et al. (2011b), PELDZIUS et al. (2011), BANHESSE et al. (2012), GARCIA-MIRELES et al. (2012), PELDZIUS et al. (2012), GARZÁS et al. (2013)), qualitativo ou quantitativo. Entre os critérios quantitativos estão: (F) Fortemente Relacionado: 86 - 100\%; (L) Largamente Relacionado: 51 - 85\%; (P) Parcialmente Relacionado: 16 - 50\%; (D) Dificilmente Relacionado: 1 - 15\%; (N) Não Relacionado.

A segunda questão secundária, "Quais são as características das iniciativas de melhorias de processos de software multimodelos em organizações?", dos artigos que apresentaram um framework de melhoria do processo (FERREIRA et al. (2011), HAUCK et al. (2011), PARDO et al. (2011b), BANHESSE et al. (2012), BUGLIONE et al. (2012), FURTADO et al. (2012), PARDO et al. (2012), PELDZIUS et al. (2012), GARZÁS et al. (2013)), as características eram as mesmas: orientado por capacidade do processo, com fases bem definidas. Para cada framework, as fases variavam bastante. Porém, uma atividade comum a todos é o mapeamento dos resultados dos processos e dos atributos de processo para realizar o mapeamento com o modelo de referência.

Os artigos mapearam ou harmonizaram processos (PELDZIUS et al. (2011), RUIZ et al. (2011), BALDASSARRE et al. (2011), GARCIA-MIRELES et al. (2012)) trataram de pelo menos uma guia de melhoria de processos, mais utilizada no mercado mundial: CMMI-DEV e ISO/IEC 15504. Um dos artigos selecionados sintetizou as características de harmonizações existentes, como: (i) União: a junção de práticas e recomendações dos modelos; (ii) Interseção: os elementos comuns entre os modelos são apresentados; (iii) Diferença: As diferenças entre os modelos são destacadas; (iv) Complemento: $\mathrm{O}$ complemento do modelo que está presente em outros é destacado.

Dez dos artigos selecionados (FERREIRA et al. (2011), HAUCK et al. (2011), PARDO et al. (2011b), BANHESSE et al. (2012), BUGLIONE et al. (2012), FURTADO et al. (2012), PARDO et al. (2012), PELDZIUS et al. (2012), GARZÁS et al. (2013), PARDO et al. (2011a)) demonstram a tendência da comunidade científica em construir frameworks para resolver o problema da utilização de multimodelos, que afeta a todas as organizações de Tecnologia da Informação. Estes artigos também demonstram como é atual o tema de harmonização de multimodelos. O restante dos artigos apresentam o mapeamento entre modelos (quatro artigos identificados) e demonstram a tendência de utilização de método e critério para a representação da aderência e rastreabilidade. Este aspecto demonstra a importância de revelar o grau de aderência de um modelo em relação ao outro, assim como suas interseções.

Com base neste mapeamento sistemático (ARAUJO, 2014) foi possível identificar que o melhor caminho para a utilização de dois modelos é a sua comparação por meio de critérios quantitativos, orientado pelos resultados dos processos e dos atributos do 
processo, para a representação da aderência e rastreabilidade. Também percebeu-se a inexistência de mapeamento entre os dois modelos alvo deste artigo: o MR-MPS-SW e o CERTICS.

\section{Modelos de Referência MR-MPS-SW e CERTICS}

O MPS.BR - Melhoria do Processo de Software Brasileiro é um programa mobilizador iniciado em 2003, coordenado pela SOFTEX (2012a), com o objetivo de melhorar a capacidade das empresas brasileiras e torná-las mais competitivas no mercado internacional. O modelo de referência MR-MPS-SW define 7 níveis de maturidade, apresentado na Tabela 2, que são uma combinação entre o processo e sua capacidade, assim como 19 processos. Os processos no MR-MPS-SW são descritos em termos de propósito e resultados esperados. O propósito descreve o objetivo geral a ser alcançado e os resultados esperados do processo estabelecem as evidências esperadas, que devem ser encontradas em produtos de trabalho produzidos pela execução do processo.

Tabela 2: Níveis de Maturidade do Modelo MR-MPS-SW

\begin{tabular}{|c|c|c|c|}
\hline \multicolumn{2}{|r|}{ NÍVEL DE MATURIDADE } & PROCESSOS & ATRIBUTOS DE PROCESSO \\
\hline A & Em Otimização & & $\begin{array}{l}\text { AP 1.1, AP 2.1, AP 2.2, AP 3.1, } \\
\text { AP 3.2, AP 4.1, AP } 4.2, \text { AP } 5.1 \text { e } \\
\text { AP 5.2 }\end{array}$ \\
\hline B & $\begin{array}{c}\text { Gerenciado } \\
\text { Quantitativamente }\end{array}$ & Gerência de Projetos (GPR) - Evolução & $\begin{array}{l}\text { AP 1.1, AP } 2.1 \text {, AP } 2.2, \text { AP } 3.1 \text { e } \\
\text { AP 3.2, AP } 4.1 \text { e AP } 4.2\end{array}$ \\
\hline C & Definido & $\begin{array}{l}\text { Gerência de Riscos (GRI) } \\
\text { Desenvolvimento para Reutilização (DRU) } \\
\text { Gerência de Decisões (GDE) }\end{array}$ & $\begin{array}{l}\text { AP 1.1, AP 2.1, AP 2.2, AP } 3.1 \mathrm{e} \\
\text { AP } 3.2\end{array}$ \\
\hline D & Largamente Definido & $\begin{array}{l}\text { Verificação (VER) } \\
\text { Validação (VAL) } \\
\text { Projeto e Construção do Produto (PCP) } \\
\text { Integração do Produto (ITP) } \\
\text { Desenvolvimento de Requisitos (DRE) }\end{array}$ & $\begin{array}{l}\text { AP 1.1, AP 2.1, AP 2.2, AP } 3.1 \text { e } \\
\text { AP } 3.2\end{array}$ \\
\hline $\mathbf{E}$ & Parcialmente Definido & $\begin{array}{l}\text { Gerência de Projetos (GPR) - Evolução } \\
\text { Gerência de Reutilização (GRU) } \\
\text { Gerência de Recursos Humanos (GRH) } \\
\text { Definição do Processo Organizacional (DFP) } \\
\text { Avaliação e Melhoria do Processo } \\
\text { Organizacional (AMP) }\end{array}$ & $\begin{array}{l}\text { AP 1.1, AP 2.1, AP 2.2, AP } 3.1 \mathrm{e} \\
\text { AP } 3.2\end{array}$ \\
\hline $\mathbf{F}$ & Gerenciado & $\begin{array}{l}\text { Medição (MED) } \\
\text { Garantia da Qualidade (GQA) } \\
\text { Gerência de Portfólio de Projetos (GPP) } \\
\text { Gerência de Configuração (GCO) } \\
\text { Aquisição (AQU) }\end{array}$ & AP 1.1, AP 2.1 e AP 2.2 \\
\hline G & $\begin{array}{l}\text { Parcialmente } \\
\text { Gerenciado }\end{array}$ & $\begin{array}{l}\text { Gerência de Requisitos (GRE) } \\
\text { Gerência de Projetos (GPR) }\end{array}$ & AP 1.1 e AP 2.1 \\
\hline
\end{tabular}

A capacidade do processo é representada por um conjunto de Atributos de Processo (AP) descritos em termos de Resultados dos Atributos de Processos (RAPs), que evoluem de acordo com o nível de maturidade. Quanto maior a maturidade, um maior nível de capacidade para desempenhar o processo deve ser alcançado (SOFTEX, 2012a).

O CERTICS (CTI Renato Archer, 2013) é um modelo de referência para certificação de software desenvolvido pelo Centro de Tecnologia da Informação Renato Acher por solicitação do Ministério da Ciência, Tecnologia e Inovação (CTI Renato Archer, 2013) com o objetivo de verificar se o software avaliado é resultante de 
desenvolvimento e inovação tecnológica realizado no país.

O Modelo de Referência está organizado em quatro camadas, que formam uma estrutura lógica top-down. A primeira camada é formada pelo conceito fundamental de software resultante de desenvolvimento e inovação tecnológica realizados no País. A segunda camada é composta por quatro áreas de competência: (i) Desenvolvimento Tecnológico (DES); (ii) Gestão de Tecnologia (TEC); (iii) Gestão de Negócios (GNE) e (iv) Melhoria Contínua (MEC). A terceira camada é composta por 16 resultados esperados que detalhem as áreas de competência. A quarta camada é composta por um conjunto de orientações e indicadores, que detalham os resultados. A Figura 1 apresenta as quatro áreas de competência do Modelo CERTICS e seus resultados esperados.

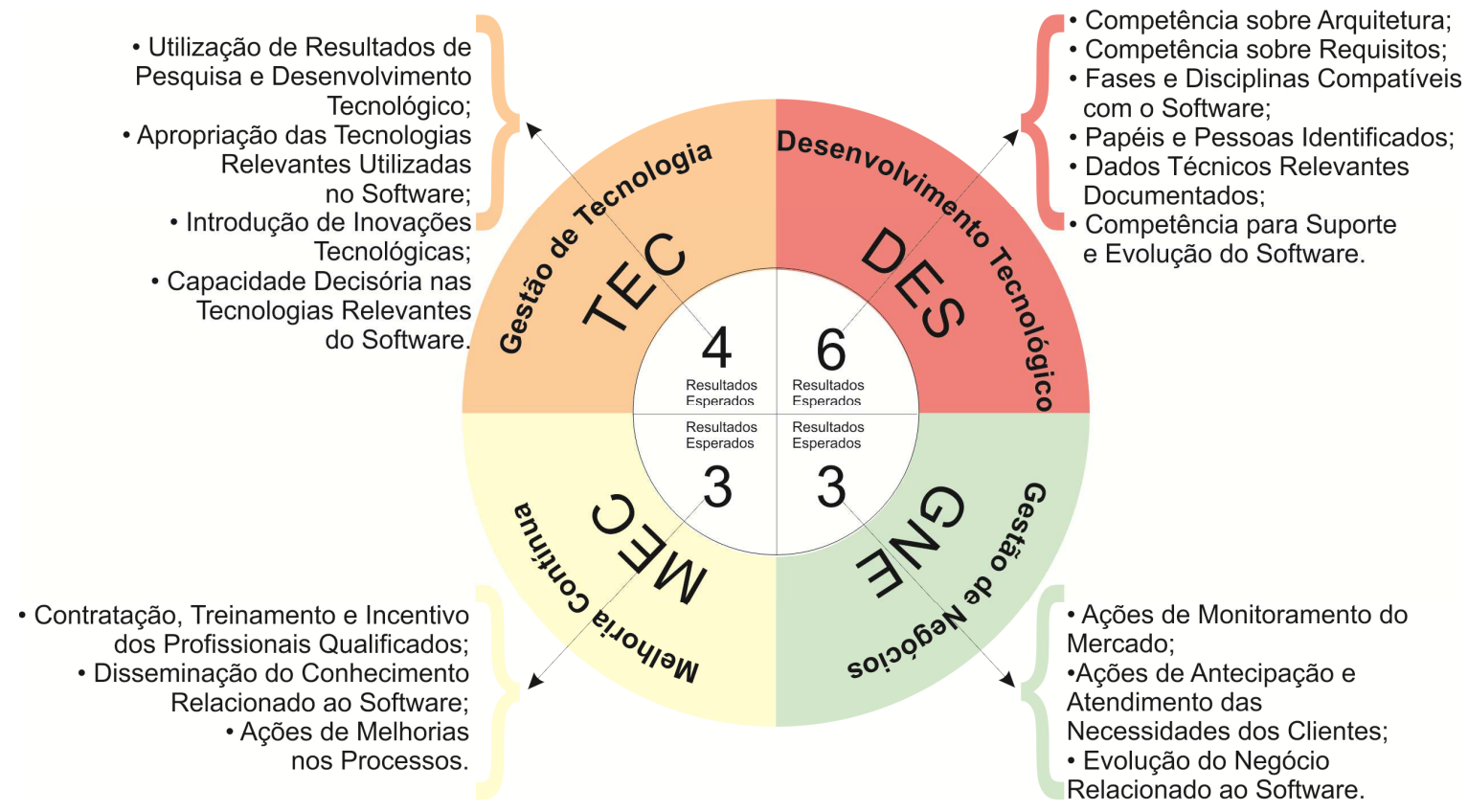

Figura 1: Áreas de Competência do Modelo CERTICS

\section{Metodologia de Pesquisa}

De acordo com BALDASSARRE et al. (2010), muitos estudos de mapeamento já foram realizados, porém sem que se definisse qual o processo utilizado para realizá-los, tornando-o não replicável. MELLO (2011) propôs uma metodologia para o mapeamento entre dois modelos, utilizando conceitos replicáveis em outros estudos. Este trabalho utilizou a metodologia definida por MELLO com pequenas adaptações para melhor adequação às características específicas deste mapeamento. A metodologia utilizada englobou as seguintes etapas.

- Revisão da Literatura: teve por objetivo identificar o estado da arte em harmonização multimodelos e realizar um estudo detalhado dos modelos envolvidos neste estudo (MR-MPS-SW e CERTICS).

- Elaboração do Mapeamento dos Modelos: teve por objetivo definir uma metodologia para a realização do mapeamento do modelo de referência MR-MPSSW (SOFTEX, 2012a) com o modelo de Certificação de Software CERTICS (CTI Renato Archer, 2013), conforme apresentada na Figura 2. 


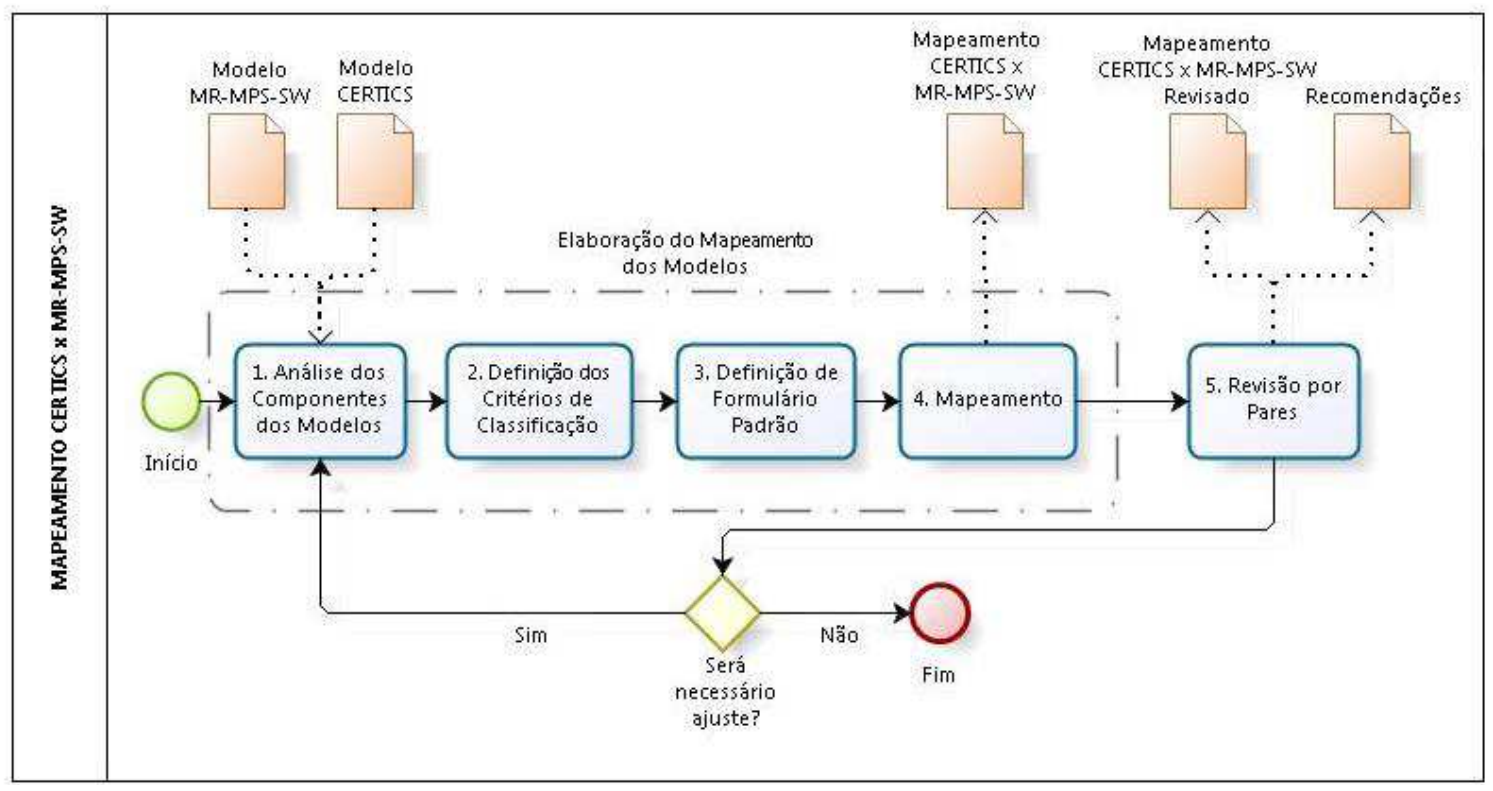

Figura 2: Mapeamento CERTICS x MR-MPS-SW

O mapeamento dos modelos define um processo com cinco atividades:

- Análise dos Componentes dos Modelos, que consiste em uma análise dos modelos a serem mapeados. O objetivo é entender cada modelo com sua estrutura, identificando seus processos e requisitos, para a adequada interpretação e comparação. Com base nos componentes identificados é definida a forma de comparação, assim como os componentes do modelo que serão excluídos do mapeamento, com as respectivas justificativas.

- Definição dos critérios de classificação, que têm como objetivo permitir que os componentes dos modelos considerados no mapeamento sejam comparados de forma tão clara quanto possível. Para que esta atividade se torne clara e padronizada devem ser definidos critérios de classificação. Além disso, ao se realizar o mapeamento, além da aplicação do critério na comparação é incluída uma observação com a justificativa da classificação.

- Definição de formulário padrão que permita descrever os componentes dos modelos sendo comparada, a indicação da equivalência ou não e as considerações associadas em um único instrumento.

- Mapeamento, que é a atividade principal e onde os resultados esperados de um modelo serão comparados com os resultados esperados do segundo modelo com o objetivo de se identificar as interseções e as diferenças.

- Revisão por Pares, cujo objetivo é obter-se uma crítica qualificada sobre o mapeamento, que permita corrigir as inadequações encontradas, a ser conduzida por especialistas nos modelos considerados. Os autores do mapeamento tinham grande experiência no modelo e de implementação do CERTICS (Mocny et al., 2014).

\subsection{Mapeamento MR-MPS-SW e CERTICS}

Na etapa "Análise dos Componentes dos Modelos" foi identificada que a certificação CERTICS está organizada em áreas de competência, cada uma delas com seus respectivos resultados esperados, com um nível de maturidade. O CERTICS não possui atributos de processo. As quatro áreas de competência são: Desenvolvimento 
Tecnológico; Gestão da Tecnologia; Gestão do Negócio; Melhoria Contínua.

O modelo MR-MPS-SW está organizado em 19 processos cada um com seus resultados esperados e em atributos de processo com seus resultados esperados. Os processos estão organizados em sete níveis de maturidade. Nesta análise foi observado que alguns dos resultados esperados das áreas de competência do CERTICS podem ser mapeados com os resultados esperados de atributos de processo no MR-MPS-SW.

De acordo com BALDASSARRE et al. (2010) para realizar o mapeamento é necessário definir um modelo de origem e um modelo de destino. O CERTICS foi escolhido como o modelo de origem e o MR-MPS-SW como o modelo de destino (CERTICS $\rightarrow$ MR-MPS-SW) na comparação, pois foi necessário comparar cada resultado esperado do CERTICS com resultados esperados dos processos do MR-MPSSW, para cada nível de maturidade.

O mapeamento "CERTICS $\rightarrow$ MR-MPS-SW" possibilita que cada resultado esperado do CERTICS seja identificado por um ou mais critérios de cobertura, a um conjunto de resultados esperados do MPS-SW, incrementado por nível de implementação. Uma diretriz orienta o que deve ser implementado a mais, para complementar a cobertura.

Como forma de obter uma comparação clara e padronizada, foi definido o seguinte critério de classificação para o mapeamento entre os modelos:

(i) COB: Coberto: O MR-MPS-SW cobre todas as exigências do resultado esperado pelo CERTICS.

(ii) COB-: Parcialmente Coberto. O MR-MPS-SW cobre alguns ou vários aspectos do resultado esperado pelo CERTICS.

(iii) NÃO: Não Coberto. O MR-MPS-SW não cobre o resultado esperado do CERTICS.

Para manter a padronização foi definido um formulário padrão para a realização do mapeamento com o objetivo de organizar adequadamente o trabalho, além de melhor identificar os modelos e seus respectivos resultados esperados. O modelo apresentado na Tabela 3 foi definido para ser utilizado na comparação entre os resultados esperados por áreas de competências do CERTICS com os resultados esperados de processos MRMPS-SW por níveis de maturidade. No formulário as descrições dos objetivos dos resultados esperados por área de competência do CERTICS serão destacadas seguindo as respectivas declarações no modelo. Estas definições são importantes por conter a explicação dos resultados esperados. As orientações do modelo CERTICS também são destacadas por resultado esperado da área de competência.

Tabela 3: Modelo de Formulário para Comparação do CERTICS com o MR-MPS-SW

\begin{tabular}{|c|c|c|c|c|}
\hline \multicolumn{5}{|c|}{ CERTICS • [ÁREA DE COMPETÊNCIA] [SIGLA] X MR-MPS-SW } \\
\hline CERTICS & & MR-MPS-SW & $\begin{array}{l}\text { Cobertura } \\
\text { do MPS }\end{array}$ & $\begin{array}{l}\text { Conside- } \\
\text { rações }\end{array}$ \\
\hline \multirow{7}{*}{$\begin{array}{l}\text { [SIGLA]: [RÓTULO DA ÁREA DE } \\
\text { COMPETÊNCIA] } \\
\text { [Definição do Resultado Esperado] } \\
\text { Orientações para que este resultado } \\
\text { esperado seja atendido: } \\
\text { (i) [Detalhamento do Resultado } \\
\text { esperado, por tópico esperado]; } \\
\text { (ii) [..]. }\end{array}$} & G & [Resultado esperado de processo atendido por nível] & \multirow{7}{*}{$\begin{array}{c}\text { [Cobertura } \\
\text { atendida por } \\
\text { nível] }\end{array}$} & \multirow{7}{*}{$\begin{array}{c}\text { [Considera- } \\
\text { ções do } \\
\text { Mapea- } \\
\text { mento]. }\end{array}$} \\
\hline & $\mathbf{F}$ & [Resultado esperado de processo atendido por nível] & & \\
\hline & $E$ & [Resultado esperado de processo atendido por nível] & & \\
\hline & D & [Resultado esperado de processo atendido por nível] & & \\
\hline & C & [Resultado esperado de processo atendido por nível] & & \\
\hline & B & [Resultado esperado de processo atendido por nível] & & \\
\hline & A & [Resultado esperado de processo atendido por nível] & & \\
\hline
\end{tabular}

ARAUJO, L. L.; ROCHA, A. R; SANTOS, G.

MR-MPS-SW e CERTICS: Mapeamento e Diretrizes para a Implantação Conjunta dos Modelos

isys - Revista Brasileira de Sistemas de Informação, Rio de Janeiro, vol. 8, No. 2, p. 34-57, 2015 
Como no mapeamento um resultado esperado pelo CERTICS pode estar relacionado a um conjunto de resultados esperados do MR-MPS-SW e cada nível de Maturidade MPS pode acrescentar resultados que complementam o resultado esperado pelo CERTICS, as considerações descrevem esta evolução e fornecem diretrizes para a implantação conjunta dos dois modelos. Este mapeamento compara os resultados esperados das áreas de competência do CERTICS com resultados esperados de processos e atributos de processos do MR-MPS-SW.

\subsection{Revisão por Pares}

Ao final do mapeamento foi realizada uma revisão por pares que teve por objetivos: (i) avaliar se as áreas de competência CERTICS estavam adequadamente comparadas aos processos do MR-MPS-SW:2012; (ii) avaliar se os resultados esperados das áreas de competência do CERTICS estavam adequadamente comparados aos resultados esperados dos processos do MR-MPS-SW:2012 por nível de capacidade; (iii) avaliar se as exclusões dos processos do MR-MPS-SW:2012 do mapeamento estão adequadas e (v) avaliar se os critérios de comparação aplicados estavam adequados.

A seleção do especialista para executar esta atividade foi realizada com base na notória especialização. Foi convidado a realizar a revisão por pares um dos autores do modelo CERTICS, que também é implementador MR-MPS-SW. A revisão por pares foi realizada utilizando critérios previamente definidos utilizados nas revisões por pares realizadas pela Equipe Técnica do Modelo MPS, que são:

- TA (Técnico Alto), indicando que foi encontrado um problema em um item que, se não for alterado, comprometerá as considerações. Esta item impacta necessariamente em um mapeamento ou critério de comparação do mapeamento.

- TB (Técnico Baixo), indicando que foi encontrado um problema em um item que seria conveniente alterar. Este problema não impacta em mapeamento nem em critério de comparação do mapeamento.

- E (Editorial), indicando que foi encontrado um erro de português ou que o texto pode ser melhorado.

- Q (Questionamento), indicando que houve dúvidas quanto ao conteúdo das considerações. Estas dúvidas devem ser esclarecidas com o avaliador, podendo se transformar em um outro tipo de avaliação.

- G (Geral), indicando que o comentário é geral em relação às considerações.

Após a realização da revisão, o especialista encontrou um problema Editorial (E), dois Técnico Alto (TA), cinco Técnico Baixo e três Gerais (um TA e dois TB), conforme apresentado na Figura 3. Após a análise dos problemas identificados, todas as correções solicitadas do tipo TA, TB, E G foram realizadas. 


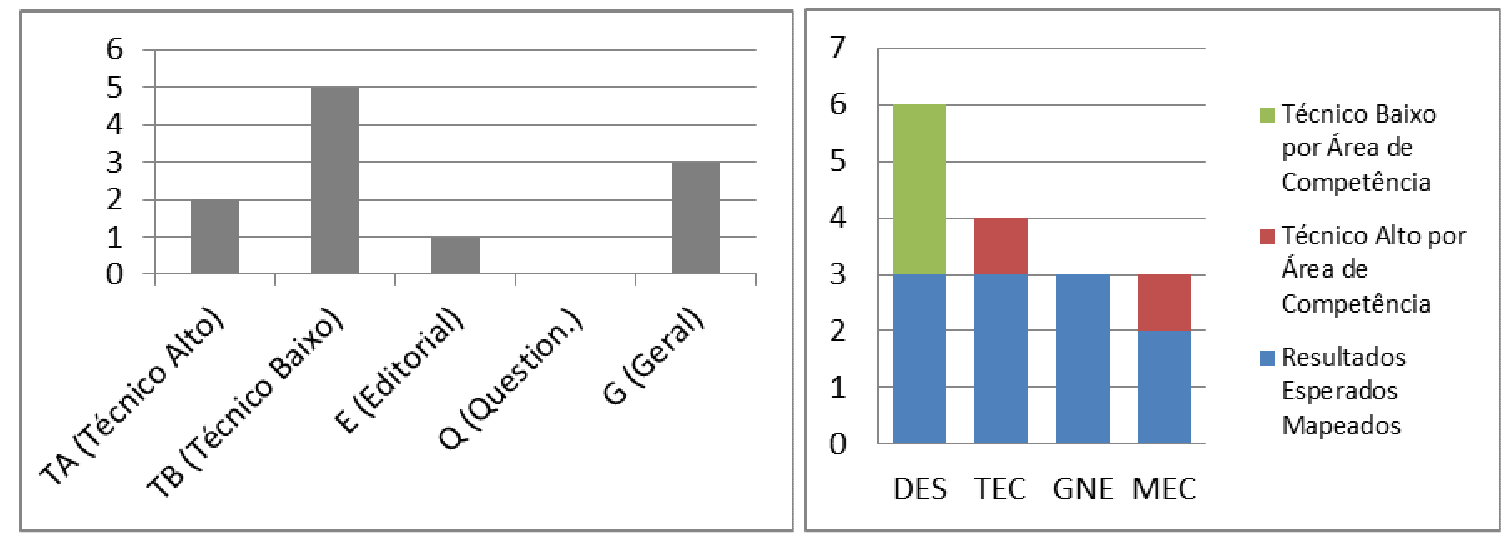

Figura 3: Problemas identificados na revisão por pares e áreas impactadas

\section{Cobertura do MR-MPS-SW com Relação ao CERTICS}

O mapeamento realizado permitiu identificar o grau de cobertura do MR-MPS-SW com relação aos resultados esperados do CERTICS. Não existe um processo do MR-MPSSW que seja equivalente a uma área de competência CERTICS, mas vários resultados esperados de processos que tratam dos resultados esperados do CERTICS, conforme apresentada na Tabela 4.

Tabela 4: Comparação dos componentes da CERTICS e MR-MPS-SW

\begin{tabular}{|c|c|c|c|c|}
\hline \multicolumn{2}{|r|}{ CERTICS } & \multicolumn{3}{|r|}{ MR-MPS-SW } \\
\hline Sigla & Área de Competência & & Sigla & Processo/Atributo de Processo \\
\hline \multirow[t]{8}{*}{ DES } & \multirow[t]{8}{*}{ Desenvolvimento Tecnológico } & G & GPR & Gerência de Projetos \\
\hline & & G & GRE & Gerência de Requisitos \\
\hline & & $F$ & GCO & Gerência de Configuração \\
\hline & & E & GPR & Gerência de Projetos Evolução \\
\hline & & E & GRH & Gerência de Recursos Humanos \\
\hline & & D & DRE & Desenvolvimento de Requisitos \\
\hline & & D & PCP & Projeto e Construção do Produto \\
\hline & & D & ITP & Integração do Produto \\
\hline \multirow[t]{2}{*}{ TEC } & \multirow[t]{2}{*}{ Gestão da Tecnologia } & G & GPR & Gerência de Projetos \\
\hline & & E & GRH & Gerência de Recursos Humanos \\
\hline \multirow[t]{5}{*}{ MEC } & \multirow[t]{5}{*}{ Melhoria Contínua } & G & GPR & Gerência de Projetos \\
\hline & & E & GRH & Gerência de Recursos Humanos \\
\hline & & E & DFP & Definição do Processo Organizacional \\
\hline & & E & AMP & Avaliação do Processo Organizacional \\
\hline & & $\mathbf{A}$ & AP 5.1 & Resultados de Atributos de Processos \\
\hline GNE & Gestão do Negócio & \multicolumn{3}{|c|}{$\begin{array}{l}\text { Não possui resultado no MPS, logo não será apresentado } \\
\text { no mapeamento abaixo. }\end{array}$} \\
\hline
\end{tabular}

A área de competência GNE (Gestão do Negócio) do modelo CERTICS não possui nenhum resultado equivalente no MR-MPS-SW. Os processos AQU (Aquisição), GQA (Garantia da Qualidade), MED (Medição), GPP (Gerência de Portfólio), GRU (Gerência de Reuso), VER (Verificação), VAL (Validação), DRU (Desenvolvimento para Reuso), GDE (Gerência de Decisão) e GRI (Gerência de Risco) do modelo MRMPS-SW não possuem equivalentes no CERTICS. 
A Tabela 5 sumariza o mapeamento realizado. Foi identificado que há $22 \%$ de cobertura do MR-MPS-SW com relação ao CERTICS. Isto ocorre porque o escopo do CERTICS não é apenas de desenvolvimento, mas permeia uma parte de $\mathrm{P} \& \mathrm{D}$, análise de mercado e manutenção de software.

Tabela 5: Resumo da Cobertura do MR-MPS-SW com relação ao CERTICS

\begin{tabular}{|c|c|c|c|c|}
\hline & DES & TEC & MEC & GNE \\
\hline 1 & COB- & NÃO & COB- & NÃO \\
\hline \multirow{2}{*}{2} & \multirow{2}{*}{ COB- } & COB- até o Nível E & NÃO até o Nível F & \multirow{2}{*}{ NÃO } \\
\hline & & COB a partir do Nível D & COB a partir do Nível E & \\
\hline \multirow{2}{*}{3} & \multirow[t]{2}{*}{ COB- } & \multirow[t]{2}{*}{ NÃO } & COB- até Nível B & \multirow[t]{2}{*}{$\overline{N A \tilde{O}}$} \\
\hline & & & COB no Nível A & \\
\hline 4 & COB- & NÃO & & \\
\hline \multirow{2}{*}{5} & COB- até o Nível E; & & & \\
\hline & COB a partir do Nível D & & & \\
\hline 6 & NÃO & & & \\
\hline
\end{tabular}

\subsection{Desenvolvimento Tecnológico (DES)}

A área de competência Desenvolvimento Tecnológico (DES) possui 6 resultados esperados que serão apresentados a seguir, com suas respectivas coberturas em relação ao MR-MPS-SW e diretrizes para a implementação conjunta.

\section{(i) DES1 • Competência sobre a Arquitetura: COB -}

Em todos os níveis do MR-MPS-SW, GPR 7 e GPR 14 (do processo Gerência de Projetos) garantem que os profissionais envolvidos no projeto sejam capazes de executar suas atividades com competência profissional.

A partir do nível E do MR-MPS-SW é implantado o processo GRH (Gerência de Recursos Humanos), onde os resultados GRH 1 e GRH 2 exigem que se identifiquem as necessidades da organização e se recrutem indivíduos com estas habilidades e competências. GRH 3 e GRH 6 tratam dos treinamentos de responsabilidade da organização e GRH 7 garante que se avalie a efetividade dos treinamentos.

Porém, é a partir do nível D do MR-MPS-SW, com o processo PCP (Projeto e Construção do Produto), que certamente as questões de DES 1 serão tratadas. Ainda no nível D, o processo ITP (Integração do Produto), nos resultados ITP 3 e ITP 4, tratam das interfaces internas e externas. A implementação de RAP 7 de ITP e PCP garante a capacitação profissional para os responsáveis da arquitetura. Entretanto, algumas exigências de DES 1 não são tratadas no MR-MPS-SW em nenhum processo ou atributo de processo.

A Tabela 6 apresenta as diretrizes para implementação conjunta do MR-MPS-SW e CERTICS relacionada ao resultado esperado DES1 do CERTICS.

Tabela 6: Diretrizes para implementação conjunta do MR-MPS-SW e CERTICS: DES1 O R I E N T A Ç Ã O

Para complementar este resultado do CERTICS, uma empresa com MR-MPS-SW deve: (i) evidenciar que os responsáveis pela arquitetura residam no país, sejam contratados CLT ou que são sócios da empresa; (ii) em caso de aquisição de componentes, ter autonomia para a tomada de decisões sobre esses componentes; (iii) evidenciar a realização de atualizações nos componentes adquiridos; (iv) caso esteja nos níveis G, F ou E, garantir que exista uma definição adequada da arquitetura. 


\section{(ii) DES 2 - Competência sobre os requisitos: COB-}

Em todos os níveis do MR-MPS-SW, o resultado esperado GPR 7 garante que os profissionais envolvidos no projeto sejam capazes de executar suas atividades com competência profissional e o GPR 9 garante que os dados relevantes do projeto, o que inclui os requisitos, estejam identificados e armazenados com controle de acesso. GPR14 garante que o planejado nestes dois resultados é implementado. Em todos os níveis MR-MPS-SW deve estar implementado o processo GRE (Gerência de Requisitos), onde os resultados GRE 5 tratam da gestão de mudanças, GRE 3 trata da rastreabilidade e GRE 4 da consistência entre os produtos de trabalho e os requisitos. Estes dois últimos resultados garantem a consistência do produto ao serem realizadas mudanças.

A partir do nível E do MR-MPS-SW é implantado o processo GRH onde os resultados GRH 1 e GRH 2 exigem que se identifiquem as necessidades da organização e se recrutem indivíduos com estas habilidades e competências. GRH 3 e GRH 6 tratam dos treinamentos de responsabilidade da organização, o que certamente deve incluir as questões tratadas em DES 1. GRH 7 garante que se avalie a efetividade dos treinamentos.

A partir do nível D do MR-MPS-SW é implantado o processo DRE. Os resultados DRE 3, DRE 4 e DRE 5 apoiam o cumprimento dos requisitos de DES 2, na definição e documentação dos requisitos. O RAP 7 deste processo garante a competência profissional das pessoas que executam o processo DRE e o RAP 13 garante que os produtos de trabalho estejam armazenados com níveis apropriados de controle.

A Tabela 7 apresenta as diretrizes para implementação conjunta do MR-MPS-SW e CERTICS relacionada ao resultado esperado DES2 do CERTICS.

Tabela 7: Diretrizes para implementação conjunta do MR-MPS-SW e CERTICS: DES2

$$
\text { O R I E N T A Ç Ã O }
$$

Para complementar este resultado do CERTICS, uma empresa com MR-MPS-SW deve: (i) evidenciar que os responsáveis pelos requisitos residam no país, sejam contratados CLT ou que seja sócios da empresa; (ii) em caso de aquisição de componentes, possuir autonomia para a tomada de decisões sobre os requisitos dos componentes; (iii) evidenciar a realização de atualizações nos requisitos dos componentes adquiridos; (iv) caso seja uma empresa com nível MPS anterior ao D, garantir que exista uma definição adequada dos requisitos.

\section{(iii) DES 3 - Fases e disciplinas compatíveis com o software: COB-}

Este resultado esperado do CERTICS é atendido, em parte, pelo nível G do MR-MPSSW onde é implementado o processo GPR. DES 3 cobre basicamente dois aspectos: que a empresa seja capaz de mostrar as fases e disciplinas realizadas para $o$ desenvolvimento (independente de que estas tenham sido planejadas) e que elas sejam compatíveis com o software. Estes dois aspectos são cobertos por GPR, pois o cronograma é considerado e deve cobrir todas as fases e disciplinas.

A Tabela 8 apresenta as diretrizes para implementação conjunta do MR-MPS-SW e CERTICS relacionada ao resultado esperado DES3 do CERTICS.

Tabela 8: Diretrizes para implementação conjunta do MR-MPS-SW e CERTICS: DES3 O R I E N T A Ç Ã O

Para complementar este resultado do CERTICS, uma empresa com MPS-SW deve garantir:

(i) que a organização tenha autonomia para atualização de componentes adquiridos e (ii) no caso de aquisição contendo tecnologia relevante, demonstrar as fases e disciplinas de uma

ARAUJO, L. L.; ROCHA, A. R; SANTOS, G.

MR-MPS-SW e CERTICS: Mapeamento e Diretrizes para a Implantação Conjunta dos Modelos

iSys - Revista Brasileira de Sistemas de Informação, Rio de Janeiro, vol. 8, No. 2, p. 34-57, 2015 
atualização relevante.

\section{(iv) DES 4 • Papéis e Pessoas Identificados: COB-}

Em todos os níveis do MR-MPS-SW, GPR 7 e GPR14 garantem que todos os profissionais envolvidos no projeto sejam capazes de executar suas atividades com competência profissional.

A partir do nível E do MR-MPS-SW é implantado o processo GRH (Gerência de Recursos Humanos) onde os resultados GRH 1 e GRH 2 exigem que se identifiquem as necessidades da organização e se recrutem indivíduos com estas habilidades e competências. GRH 3 e GRH 6 tratam dos treinamentos de responsabilidade da organização, o que certamente deveriam incluir as questões tratadas em DES 1. O GRH 7 garante que se avalie a efetividade dos treinamentos. DES 4 faz, também, referência a profissionais relacionados a negócios, suporte e evolução do software, o que não é tratado pelo MR-MPS-SW. Para o resultado esperado DES 4, o MR-MPS-SW cobre apenas alguns resultados esperados do CERTICS.

A Tabela 9 apresenta as diretrizes para implementação conjunta do MR-MPS-SW e CERTICS relacionada ao resultado esperado DES4 do CERTICS.

Tabela 9: Diretrizes para implementação conjunta do MR-MPS-SW e CERTICS: DES4 O R I E N T A Ç Ã O

Para complementar este resultado da CERTICS, uma empresa com MR-MPS-SW deve: (i) garantir que os profissionais relacionados a negócios, suporte e evolução do software estejam identificados e possuam formação, habilidades e conhecimentos adequados.

(v) DES 5 - Dados técnicos relevantes documentados: COB- até o Nível E; COB a partir do Nível D

Em todos os níveis do MR-MPR-SW, GPR 9 e GPR 14 garantem que os dados relevantes do projeto estejam armazenados e acessíveis. O processo GRE, nos resultados esperados GRE1 e GRE2, tratam da documentação dos requisitos do cliente. GRE 3 trata da documentação da rastreabilidade dos requisitos com os demais produtos de trabalho.

A partir do nível F do MR-MPS-SW é implementado o processo GCO (Gerência de Configuração) que garante o armazenamento e disponibilização dos dados do projeto de forma mais rigorosa.

A partir do nível D, a implementação dos processos DRE (Desenvolvimento de Requisitos), PCP e ITP garante o total atendimento de documentação dos dados de requisitos e arquitetura.

A Tabela 10 apresenta as diretrizes para implementação conjunta do MR-MPS-SW e CERTICS relacionada ao resultado esperado DES5 do CERTICS.

Tabela 10: Diretrizes para implementação conjunta do MR-MPS-SW e CERTICS: DES5 O R I E N T A Ç Ã O

Para complementar este resultado do CERTICS, uma empresa com MR-MPS-SW níveis G, F ou $\mathrm{E}$ deve garantir que os requisitos e a arquitetura estejam definidos e completamente documentados.

(vi) DES 6 • Competência para suporte e evolução do sistema: NÃO

O MR-MPS-SW não possui processos e resultados de atributos de processos relacionados ao resultado esperado DES 6. 


\subsection{Gestão de Tecnologia (TEC)}

A área de competência Gestão de Tecnologia (TEC) possui 4 resultados esperados que serão apresentados a seguir, com suas respectivas coberturas em relação ao MR-MPSSW e diretrizes para a implementação conjunta.

(i) TEC 1 - Utilização de resultados de pesquisa e desenvolvimento tecnológico: NÃO

O MR-MPS-SW não possui processos e resultados de atributos de processos relacionados ao resultado esperado TEC 1.

(ii) TEC 2 - Apropriação das tecnologias relevantes utilizadas no software: COBaté o Nível E; COB a partir do Nível D

Em todos os níveis do MR-MPS-SW, GPR 7 garante que os profissionais envolvidos no projeto sejam capazes de executar suas atividades com competência e GPR 9 garante que os dados relevantes do projeto estejam identificados e armazenados com controle de acesso. GPR14 garante que o planejado nestes dois resultados é implementado.

A partir do nível E do MR-MPS-SW é implantado o processo GRH onde os resultados GRH 1 e GRH 2 exigem que se identifiquem as necessidades da organização e se recrutem indivíduos com estas habilidades e competências. GRH 3 a GRH 6 tratam dos treinamentos de responsabilidade da organização, o que certamente deve incluir as questões de apropriação das tecnologias relevantes conforme requerido em TEC 2. GRH 7 garante que se avalie a efetividade dos treinamentos. GRH 9, GRH 10 e GRH 11 garantem a implantação de um Sistema de Gestão do Conhecimento, com a disponibilização e compartilhamento dos registros de gestão do conhecimento.

A partir do nível D do MR-MPS-SW, o RAP 7 garante a competência profissional das pessoas que executam o processo DRE, PCP e ITP, o que pode não estar garantido nos níveis anteriores.

A Tabela 11 apresenta as diretrizes para implementação conjunta do MR-MPS-SW e CERTICS relacionada ao resultado esperado TEC2 do CERTICS.

Tabela 11: Diretrizes para implementação conjunta do MR-MPS-SW e CERTICS: TEC2 O R I E N T A Ç Ã O

Para complementar este resultado do CERTICS, uma empresa com MR-MPS-SW deve: (i) nos níveis $G, F$ e $E$ garantir que os requisitos e a arquitetura estejam definidos e documentados e (ii) nos níveis $\mathrm{G}$ e $\mathrm{F}$ garantir, também, os resultados de GRH relacionados a treinamento organizacional e gerência do conhecimento.

\section{(iii) TEC 3 • Introdução de inovações tecnológicas: NÃO}

O MR-MPS-SW não possui processos e resultados de atributos de processos relacionados ao resultado esperado TEC 3.

\section{(iv) TEC 4 • Capacidade decisória nas tecnologias relevantes do software: NÃO}

A capacidade decisória para atualizações no caso de tecnologias relevantes adquiridas não é exigência de nenhum resultado de processo ou atributo de processo MPS. 


\subsection{Melhoria Contínua (MEC)}

A área de competência Melhoria Contínua (MEC) possui 3 resultados esperados que serão apresentados a seguir, com suas respectivas coberturas em relação ao MR-MPSSW e diretrizes para a implementação conjunta.

(i) MEC 1 - Contratação, treinamento e incentivo dos profissionais qualificados: COB-

Em todos os níveis do MR-MPS-SW, GPR 7 e GPR 14 garantem que todos os profissionais envolvidos no projeto sejam capazes de executar suas atividades com competência profissional. RAP 7 garante a competência dos profissionais para os processos executados.

A partir do nível E do MR-MPS-SW é implantado o processo GRH onde os resultados GRH 1 e GRH 2 exigem que se identifiquem as necessidades da organização e se recrutem indivíduos com estas habilidades e competências. GRH 3 a GRH 6 tratam dos treinamentos de responsabilidade da organização. GRH 7 garante que se avalie a efetividade dos treinamentos.

A Tabela 12 apresenta as diretrizes para implementação conjunta do MR-MPS-SW e CERTICS relacionada ao resultado esperado MEC2 do CERTICS.

Tabela 12: Diretrizes para implementação conjunta do MR-MPS-SW e CERTICS: MEC1 O R I E N T A Ç Ã O

Para complementar este resultado do CERTICS, uma empresa com MR-MPS-SW deve: (i) garantir que os indivíduos contratados para as atividades tecnológicas, de negócio, suporte e evolução do produto possuam as habilidades e competências necessárias ou recebam treinamentos pertinentes; (ii) que existam programas de incentivo para os funcionários.

(ii) MEC 2 - Disseminação do conhecimento relacionado ao software: NÃO até o Nível F; COB a partir do Nível E

Nos níveis $\mathrm{G}$ e $\mathrm{F}$ não há resultados do MR-MPS-SW relacionados à gerência do conhecimento. A partir do nível E é implantada a gerência do conhecimento no processo Gerência de Recursos Humanos.

A Tabela 13 apresenta as diretrizes para implementação conjunta do MR-MPS-SW e CERTICS relacionada ao resultado esperado MEC2 do CERTICS.

Tabela 13: Diretrizes para implementação conjunta do MR-MPS-SW e CERTICS: MEC2

O R I E N T A Ç Ã O

Para complementar este resultado do CERTICS, uma empresa com MR-MPS-SW níveis G e

F deve implantar algum mecanismo de gerência do conhecimento.

(iii) MEC 3: Ações de melhorias nos processos: COB- até Nível B; COB no Nível A

No nível G do MR-MPS-SW, RAP 3 garante que a execução do processo seja planejada, o que implica em que pelo menos os processos Gerência de Projetos e Gerência de Requisitos estejam documentados, o que já satisfaz MEC.3 para atividades tecnológicas.

A partir do nível E do MR-MPS-SW é implementado o processo DFP (Definição do Processo Organizacional). Com isso, para todos os processos dos níveis implementados, é definido um processo padrão com tarefas, atividades, papéis e 
produtos de trabalho. O processo AMP (Avaliação e Melhoria do Processo Organizacional) também é implementado neste nível, o que implica na existência de dados sobre a execução dos processos, identificação e implantação de melhorias. No nível E é requerida a existência de um profissional ou grupo de profissionais responsável pelos processos. Logo, a partir do nível E, com principalmente DFP e AMP, o MPS cobre mais ainda atividades tecnológicas, com mais exigências. Porém, as atividades de negócios não estão no escopo deste nível do MR-MPS-SW.

No nível A, com a RAP 35 sobre objetivos de negócio, o MR-MPS-SW cobre totalmente MEC.3.

A Tabela 14 apresenta as diretrizes para implementação conjunta do MR-MPS-SW e CERTICS relacionada ao resultado esperado MEC3 do CERTICS.

Tabela 14: Diretrizes para implementação conjunta do MR-MPS-SW e CERTICS: MEC3 O R I E N T A Ç Ã O

Para complementar este resultado do CERTICS, uma empresa com MR-MPS-SW níveis G ao B deve garantir que as melhorias relativas ao objetivo de negócio sejam implementadas.

\subsection{Limitações e Ameaças}

Este trabalho possui algumas limitações. Embora os mapeamentos tenham sido avaliados por meio de revisão por pares de especialistas, estes não foram ainda avaliados em situação real. Outra limitação é o fato de só ter sido realizado o mapeamento em um sentido, do CERTICS para o MR-MPS-SW.

A limitação de se fazer o mapeamento no sentido do MR-MPS-SW para o CERTICS se deve ao fato dos modelos terem a forma de avaliação diferenciada: o modelo CERTICS é booleano (tem-se a certificação ou não). O modelo MR-MPS-SW é avaliado por níveis de maturidade (são 7 níveis), onde quanto maior o nível, maior a sua exigência, onde novos processos são incluídos. Desta forma, para se comparar cada resultado esperado do MR-MPS-SW em relação ao CERTICS seria necessário fazer esta analisar para cada nível de implementação. Esta forma seria muito complexa para consulta futura.

Outro ponto importante a ser abordado é o escopo da certificação. O CERTICS é uma avaliação de software, o MPS-SW é uma avaliação de empresa. Por isto, os critérios utilizados na comparação verifica a cobertura do MR-MPS-SW em relação a cada resultado esperado do CERTICS, o que mais uma vez orienta o sentido escolhido por este mapeamento.

As ameaças foram tratadas para evitar seus riscos. Uma ameaça importante está na revisão por um único especialista em cada modelo. A escolha dos especialistas com notória especialização foi realizada para reduzir esta ameaça.

Outra ameaça é o fato do resultado deste mapeamento MPS-SW com CERTICS não ter sido implantado em uma situação real. Para tratar este risco foi realizada a revisão por pares por especialista, visando evitar erros de entendimento.

\section{Conclusão}

Organizações realizam investimentos em melhoria de processos de software com o propósito de alcançar maior qualidade em seus produtos, além de obter as certificações requeridas. Existem atualmente uma multiplicidade de modelos que tornam difícil a 
escolha de que modelos implementar e de como implementá-los em conjunto quando necessário. Este artigo descreve o mapeamento para harmonização dos modelos MRMPS-SW e CERTICS.

Foi realizado um mapeamento sistemático da literatura que identificou o crescimento contínuo de publicações com a preocupação de harmonização de modelos de qualidade. Este estudo mostrou a importância da realização de mapeamentos entre modelos como uma ferramenta de apoio à indústria de TI na implantação de multimodelos.

Após a realização do mapeamento dos modelos CERTICS e MR-MPS-SW, foi possível observar $22 \%$ de cobertura do MR-MPS-SW com relação ao CERTICS. As orientações que devem ser seguidas ao longo da implantação do CERTICS estão descritas neste artigo, considerando o nível de capacidade MR-MPS-SW. O mapeamento nos dois sentidos seria possível, porém seria necessário um conjunto de resultados esperados do MPS para atender a um resultado do CERTICS, logo, uma visualização de processos diferentes com vários resultados específicos para representar a cobertura de um único resultado do CERTICS.

A vantagem da utilização deste mapeamento é facilitar a implantação do Modelo CERTICS em um software construído por uma Empresa com a implementação MRMPS-SW, a partir das suas respectivas diretrizes. O MR-MPS-SW possui 10 anos, já está consolidado e com possui muitos consultores e empresas implementadoras. $\mathrm{O}$ CERTICS possui 2 anos e poucos consultores. Esta referência pode ajudar aos implementadores do MR-MPS-SW a orientar a certificação de software com o modelo CERTICS, em empresas já implementadas MR-MPS-SW, em qualquer nível de maturidade.

O resultado do mapeamento fica mais completo de acordo com o nível implantado. O mapeamento apresentado pode nortear: (i) a implantação do MR-MPSSW em organizações que visem, também, à certificação CERTICS; (ii) para organizações que já possuem o MR-MPS-SW e estão se preparando para a certificação CERTICS; (iii) nortear a implantação conjunta destes modelos, evitando as redundâncias de atividades e processos.

Como trabalho futuro, pretende-se realizar um estudo de caso em uma organização que já possua o MR-MPS-SW e deseje certificar o software produzido no CERTICS para identificar se o mapeamento proposto é de fácil utilização.

\section{Agradecimentos}

Os autores agradecem a Clênio Salviano pela revisão por pares do mapeamento e a Marcelo Thiry, Adriano Albuquerque e Geraldo Xexéo pelas contribuições ao trabalho.

\section{Referências}

ARAUJO, L., 2014. "Mapeamento do MPS.SW com os Modelos MPT.BR e Certics". Dissertação de M.Sc., COPPE/UFRJ, Rio de Janeiro - RJ, Brasil.

ARAUJO, L., ROCHA, A. R., SANTOS, G., 2014. "Mapeamento dos Modelos MR-MPS-SW e CERTICS para a Implantação Conjunta". Workshop Anual do MPS - WAMPS 2015. Campinas - SP, pp 28-38.

BALDASSARRE, M. T.; CAIVANO, D.; PINO, F. J.; PIATTINI, M.; VISAGGIO, G., 2010. "A Strategy for Painless Harmonization of Quality Standards: A Real Case". In: ProductFocused Software Process Improvement, vol. 6156, Springer Science + Business Media, pp 
395-408.

BALDASSARRE, M., CAIVANO, D., PINO, F. J., PIATTINI, M., \& VISAGGIO, G., 2011. "Harmonization of ISO/IEC 9001:2000 and CMMI-DEV from a theoretical comparison to a real case application". In: Software Quality Journal, vol. 20, Springer Science + Business Media, pp 309-335.

BANHESSE, E. L., SALVIANO, C. F., JINO, M., 2012. "Towards a Metamodel for Integrating Multiple Models for Process Improvement". Software Engineering and Advanced Applications (SEAA), 2012 38th EUROMICRO Conference on. Cesme, Izmir, IEEE, 315 318.

BUGLIONE, L.; HAUCK, J. C. R., WANGENHEIM, C. G. V., CAFFERY, F. MC., 2012. "Hybriding CMMI and Requirement Engineering Maturity \& Capability Models". 7th International Conference on Software Paradigm Trends. Rome, Italy.

CATER-STEEL, A. AND TAN, W.-G. AND TOLEMAN, M., 2006. "Challenge of adopting multiple process improvement frameworks". In: 14th European Conference on Information Systems (ECIS 2006), 12-14 June, Goteborg, Sweden.

CTI Renato Archer, 2013. "Modelo de Referência para Avaliação da CERTICS - Documento de Detalhamento". Versão 1.1. Relatório Técnico CTI Renato Archer - TRT0084113.

CTI Renato Archer, 2015. Site: http://www.certics.cti.gov.br/?page id=129. Acessado em 18/03/2015.

FERREIRA, A. L., MACHADO, R. J., \& PAULK, M. C., 2011. "Supporting Audits and Assessments in Multi-model Environments". In: Product-Focused Software Process Improvement, vol. 6759, Springer-Verlag Berlin Heidelberg, pp 73-87.

FURTADO, J. C., OLIVEIRA, R. B., 2012. "A Process Framework fot the Software and Related Services Acquisition Based on the CMMI-ACQ and the MPS.BR Acquisition Guide.”. In: Latin America Transactions, IEEE (Revista IEEE America Latina), vol. 10, issue 5, IEEE, pp 2256 - 2262

GARCÍA-MIRELES, G. A., MORAGA, M. Á., GARCÍA, F., PIATTINI, M., 2012. "Towards the Harmonization of Process and Product Oriented Software Quality Approaches". In: Systems, Software and Services Process Improvement, vol. 301, Springer-Verlag Berlin Heidelberg, pp 133-144.

GARZÁS, J., PINO, FRANCISCO J., PIATTINI, M.; FERNANDEZ, C. M., 2013. “A Maturity Model for the Spanish Software Industry based on ISO Standards". In: Computer Standards \& Interfaces, vol. 35, issue 6, Elsevier, pp 616-618.

HAUCK, J. C., WANGENHEIM, C. G. V., CAFFERY, F. MC; BUGLIONE, L., 2011. "Proposing an ISO/IEC 15504-2 Compliant Method for Process Capability/Maturity Models Customization". In: Product-Focused Software Process Improvement, vol. 6759, SpringerVerlag Berlin Heidelberg, pp 44-58.

ISO/IEC, 2004. "ISO/IEC 15504-2 Software engineering — Process assessment - Part 2: Performing an assessment". In: ISO/IEC.

ISO/IEC, 2008a. "ISO/IEC 12207 Systems and Software Engineering - Software Life Cicle Process". In: ISO/IEC.

ISO/IEC, 2008b. "ISO/IEC 9001:2008 Quality Management System - Requirements.". In: ISO/IEC.

itSMF UK, 2012. “An Introductory Overview of ITIL® 2011”. $1^{\text {a }}$ ed. London, TSO Information e Publishing Solution.

KELEMEN, Z. D., 2013. "Process Based Unification for Multi-Model Software Process Improvement". D.Sc., Eindhoven University of Technology, Budapest, Hungary. 
MELLO, M. S., 2011. "Melhoria de Processos de Software Multi-Modelos Baseada nos Modelos MPS e CMMI-DEV”. Dissertação de M.Sc., COPPE/UFRJ, Rio de Janeiro, RJ, Brasil.

MOCNY, E., ARAUJO, L., MONTONI, M., IRIGOYEN, A., 2014. "Relato de Experiência da Certificação do software PRIME Saúde da ECO Sistemas na CERTICS". SBQS - Simpósio Brasileiro de Qualidade de Software. Blumenau - Santa Catarina. Outubro.

PARDO, C., PINO, F. J., GARCÍA, F., VELTHIUS, M. P., \& BALDASSARRE, M. T., 2011a. "Trends in Harmonization of Multiple Reference Models". In: Evaluation of Novel Approaches to Software Engineering, vol. 230, Springer Berlin Heidelberg, pp. 61-73.

PARDO, C.; PINO, F. J.; PIATTINI, M.; BALDASSARRE, M. T., 2011b. "Supporting the Combination and Integration of Multiple Standards and Models". Computing Congress (CCC), 2011 6th. Colombian, pp 1-6.

PARDO, C., PINO, F. J., GARCÍA, F., PIATTINI, M., \& BALDASSARRE, M. T., 2012. “An Ontology for the Harmonization of Multiple Standards and Models". In: Computer Standards \& Interfaces, vol. 34, Elsevier B.V, pp 48-59.

PELDZIUS, S., \& RAGAISIS, S., 2011. "Comparison of Maturity Levels in CMMI-DEV and ISO/IEC 15504". In: AMERICAN-MATH'11/CEA'11 Proceedings of the 2011 American conference on applied mathematics and the 5th WSEAS international conference on Computer engineering and applications, pp 117-122, January.

PELDZIUS, S., \& RAGAISIS, S., 2012. "Framework for Usage of Multiple Software Process Models". In: Software Process Improvement and Capability Determination, vol. 290, Springer Berlin Heidelberg, pp 201-221.

PINO, F. J., BALDASSARRE, M. T., PIATTINI, M., VISAGGIO, G., 2009. "Harmonizing maturity levels from CMMI-DEV and ISO/IEC 15504". In: Journal of Software Maintenance and Evolution: Research and Pratice, vol. 22, pp 279-296, June.

RUIZ, J. C., OSORIO, Z. B.; MEJIA, J.; MUÑOZ, M.; CHAVEZ, A. M.; OLIVARES, B. A. 2011. "Definition of a hybrid measurement process for the models ISO/IEC 15504 ISO/IEC 12207:2008 and CMMI Dev 1.3 in SMEs". IEEE Electronics, Robotics and Automotive Mechanics Conference (CERMA). Cuernavaca, Morelos, pp 421 - 426.

SEI, 2010. "CMMI for Development (CMMI-DEV)", Version 1.3, Pittsburgh, PA, Software Engineering Institute - Carnegie Mellon University.

SIVIY, J., KIRWAN, P., MARINO, L., MORLEY, J., 2008. "The Value of Harmonizing Multiple Improvement Technologies: A Process Improvement Professional's View". Software Engineering Institute, Carnegie Mellon.

SOFTEX, 2012a. "Guia Geral MPS de Software". Campinas, Sociedade SOFTEX.

SOFTEX, 2012b. "Guia de Implementação Parte 12: Análise da Aderência do MR-MPSSW:2012 em relação à NBR ISO/IEC 29110-4-1:2012 - Engenharia de Software - Perfis de ciclo de vida para micro organizações (VSEs) - Parte 4-1: Especificações de perfil: Grupo Perfil Genérico". Campinas. Sociedade SOFTEX.

SOFTEX, 2012c. "Guia de Implementação Parte 13: Mapeamento e Sistemas de Equivalências entre o MR-MPS-SW:2012 e o MoProSoft:2005". Campinas. Sociedade SOFTEX.

SOFTEX, 2015. Site: http://www.softex.br/mpsbr/avaliacoes/mps-sw/mpsbr-ma-mps/. Acessado em 18/03/2015.

SOFTEX RECIFE, 2011. "Guia de Referência do Modelo - MPT.Br". Recife. Sociedade SOFTEX RECIFE.

TENNANT, G., 2001. "Six Sigma: SPC and TQM in Manufacturing and Services". Burlington - England. Gower Publishing. 